\title{
GALVANIZED STEEL PRE-TREATED WITH HYBRID FILM CURED BY UV: INFLUENCE OF PHOTOINITIATOR CONCENTRATION ON THE CORROSION RESISTANCE
}

\author{
SANDRA KUNST ${ }^{* a}$, ELEN LEAL DA SILVA ${ }^{a}$, LEONARDO ANTONINI ${ }^{a}$, JOSE MATOS ${ }^{a}$, \\ CLAUDIA OLIVEIRA ${ }^{b}$ AND CELIA MALFATTI ${ }^{a}$
}

\author{
${ }^{a}$ Metallurgy Eng. Department, University of Federal of Rio Grande do Sul, Porto Alegre, Brazil. \\ ${ }^{b}$ Engineering Department ICET, University of Feevale, Porto Alegre, Brazil
}

(Received: May 4, 2012 - Accepted: June 26, 2012)

\begin{abstract}
Several coatings have been proposed as an alternative to replacement processes based on hexavalent chromium. Among them stand out the hybrid films. Some hybrids films, with modified composition by the addition of photoinitiators, can be cured by ultraviolet system (UV). The UV cure is the instantaneous conversion of a reactive liquid in a solid induced by radiation, starting from the polymerization and cross-linking reactions of the chemical species. In order that reaction takes place through the action of UV radiation, it is necessary to add, in the composition of the hybrid film, a substance sensitive to this radiation called photoinitiator. The aim of this work is the preparation and characterization of hybrid films, cured by UV, from TEOS and TMSPMA alkoxide precursors, with addition of photoinitiators with different concentrations (1 and 2\%) and without photoinitiator on galvanized steel. The films were characterized concerning the morphology, physicochemical properties and electrochemical behavior. The presence and the increase of photoinitiator concentration on hybrid films improved corrosion resistance of the system.
\end{abstract}

Keywords: Galvanized steel, photoinitiator, corrosion

\section{INTRODUCTION}

The curing technology by ultraviolet radiation is being increasingly used by the industrial sector furniture, metal graphic, graphic, metal mechanic, footwear among others due to its numerous advantages such as speed in the process and the attainment of a coating with excellent chemical and physical properties (Yamasaki, 1997).

Furthermore, the process can easily be automated in a sequence of operations and the main advantage of this technology is the fact that the process is environmentally friendly, since in these radiation curing systems the volatile product is part of the reaction and become part of the coating, assuming that the evaporation loss is not more than $5 \%$ (Stowe, 2000).

However, one of the main problems of the curing by ultraviolet radiation is the fact that most coatings cured by radiation presented disabled properties when applied directly on metal surfaces. This deficiency is mainly related to poor adherence and contraction of the film after curing (Pietschmann, 2005), requiring a conventional surface pre-treatment of cure by thermal system and using formulations with high levels of solvent emissions of volatile organic compounds (VOCs), that pollute the atmospheric air through photochemical reaction with nitrogen oxides (NOx) to produce ozone low-level (Roose, 2009). Besides, industries have been used the chromatization process in some nonferrous metals of difficult adhesion. However, the chromatization process, even though functional, produces toxic and carcinogenic residue to the environment (EPA, 2010).

For this reason the hybrid films have been proposed as an alternative to replacement of chromium hexavalent-based processes. In addition, some hybrid films, that composition is modified, can be cured by the curing system for ultraviolet (UV). Curing by ultraviolet radiation (UV) is the instantaneous conversion of a reactive liquid in a solid, induced by radiation. When this liquid is exposed to radiation, polymerization and cross-linking reactions started between the chemical species (Fazenda, 2005). In order that reaction takes place through the action of UV radiation, it is necessary to add a substance to the composition of the hybrid film, that is sensitive to such radiation, called photoinitiator (Graen, 2003).

The photoinitiator is the molecule responsible for the absorption of UV radiation and that, through cure mechanism by free radical, starts polymerization reaction. The UV light should penetrate the hybrid film to be absorbed by the photoinitiator. To make it happen, two conditions are necessary: the ultraviolet light must have enough energy, and the coating should not be opaque to such radiation (Lin, 2003).

The photoinitiator is a substance or mixture of substances that, by absorption of radiant energy, undergoes chemical mutation, resulting in reactive intermediates that can start a polymerization process. The concentration of the photoinitiator should be small so that the resulting products from photoreaction do not affect the quality of the coating (Rahn, 2003). In this context, the objective of this work is the preparation and characterization of hybrid films from alkoxide precursors TMSPMA and TEOS with addition of photoinitiators with different concentrations $(1$ and $2 \%$ ) and without photoinitiator on galvanized steel. The films were cured by the process of UV radiation.

\section{MATERIALS AND METHODS}

\section{Surface Preparation}

The galvanized steel substrates were washed with ethanol and dried under warm air. They were degreased with a neutral detergent $(\mathrm{pH}=7)$, at $70^{\circ} \mathrm{C}$ by immersion for 10 minutes. After that, they were washed with deionized water, dried, washed with ethanol and dried under warm air.

\section{Preparation of hybrid films}

The hydrolysis reactions of the hybrid film were carried out by alkoxide precursors (TMSPMA) -3 - (trimetoxysililpropil) methacrylate $\left(\mathrm{C}_{10} \mathrm{H}_{20} \mathrm{SiO}_{5}\right)$ and (TEOS) tetraethoxysilane $\left(\mathrm{C}_{8} \mathrm{H}_{20} \mathrm{SiO}_{4}\right)$ and conducted in solutions TEOS/ TMSPMA/alcohol/water with addition of $0.01 \mathrm{M}$ cerium nitrate. For the UV curing process, it was used samples with and without photoinitiator. The photoinitiator $\alpha, \alpha$-dimethoxy- $\alpha$-hydroxyacetophenone, that has $\mathrm{UV}$ absorption of 245-331 nm, was used in concentration of 1 and $2 \%$. After 24 hours of hydrolysis, the solution presented a final $\mathrm{pH}$ of 4.75 .

\section{Application and curing of hybrid films}

The application of solutions containing the hydrolyzed hybrid films was made by dip-coating process, with removal rate of $20 \mathrm{~cm} \cdot \mathrm{min}^{-1}$ and with time remained of 2 minutes in the solution. After the process of dip-coating, the substrates pretreated with the hybrid films were cured by ultraviolet radiation, with a treadmill speed of $9.7 \mathrm{~m} \cdot \mathrm{min}^{-1}$ and an intensity of $146 \mathrm{~mJ} . \mathrm{cm}^{2}$ with a mercury lamp of medium pressure Gallium doped.

Mercury lamps of medium pressure are commonly used in curing by UV radiation. Its power ranges between 40 to 120 Watts $/ \mathrm{cm}$ and the average pressure is 100 Torr. This lamp produces a broad range light spectrum of the $200-400 \mathrm{~nm}$ and it was chosen because its absorption in the wavelength of the photoinitiator is indicated for coatings with low thickness and not pigmented as used in this study. Metallic halide lamps were also used to cure the studied films hybrid. These are obtained by the introduction of volatile metallic halide (Gallium) in mixture of mercury, and are called doped. The effect of such additives is to shift the spectrum to bigger wavelengths.

Description of the samples

Table 1 shows the description of the samples used in this work. 
Table 1. Description of the samples.

\begin{tabular}{|c|c|}
\hline Sample & Description \\
\hline AG & Uncoated galvanized steel. \\
\hline MTSF & $\begin{array}{c}\text { Galvanized steel coated with hybrid film without } \\
\text { photoinitiator and UV cured. }\end{array}$ \\
\hline MT1F & $\begin{array}{c}\text { Galvanized steel coated with hybrid film - 1\% } \\
\text { photoinitiator and UV cured. }\end{array}$ \\
\hline MT2F & $\begin{array}{c}\text { Galvanized steel coated with hybrid film }-2 \% \\
\text { photoinitiator and UV cured. }\end{array}$ \\
\hline
\end{tabular}

\section{Characterization of hybrid films}

\section{Morphological characterization}

The morphology of the coatings was evaluated using Scanning Electron Microscopy (SEM), JEOL 6060 microscope with computer system of image acquisition. The surface morphology of the samples was observed in top view and thickness of the obtained pre-treatment was observed by the crosssectional view.

Wettabiliy

An important property of metal coated with hybrid films is its surface energy. This property is measured by contact angle $\theta$, which is an important tool to characterize the wettability of solids, in other words, the degree of hydrophobicity. The test was performed in an apparatus developed by the Laboratory of Optics \& Laser of Physic's Institute, Federal University of Rio Grande do Sul (UFRGS). In the sessile drop method, a drop of liquid properly purified is deposited on the surface of a solid with a micro syringe. The drop is observed by means of a lens of low magnification, and the contact angle measured by a goniometer.

\section{Infrared Spectroscopy}

The infrared spectroscopy measurements were performed in Laboratory of Design and Material Selection from UFRGS using a spectrophotometer Spectrum ASCII 100. The measurements were performed with the beam in the mid-infrared $\left(4000-650 \mathrm{~cm}^{-1}\right)$. The spectra were obtained from the films without a substrate (free-standing films). For this characterization, a solution containing the precursors alkoxide 3 - (trimetoxysililpropil) methacrylate (TMSPMA) and tetraethoxysilane (TEOS) with addition of $0.01 \mathrm{~mol} \mathrm{~L}^{-1}$ cerium nitrate with and without addition of the photoinitiator was used. The concentration of the photoinitiator used was 1 and $2 \%$. The solutions with and without photoinitiator were placed on a Petri plate and cured by the UV process. After obtaining the film, a small amount of each system was taken for analysis.

\section{Open circuit potential and potentiostatic polarization}

The electrochemical potentiostatic polarization test was performed with a potencistat/galvanostat Omnimetra MOD. PG-05 and a conventional cell of three electrodes. The reference electrode was saturated calomel (SCE) and counter electrode was made of platinum. The measurement was performed in an environment without agitation, aerated and at room temperature. The electrolyte was $5 \% \mathrm{NaCl}$. The open circuit potential was monitored during the first hour of immersion in the electrolyte and before potentiostatic polarization. The scan range was from $-200 \mathrm{mV}$ below the open circuit potential to 300 $\mathrm{mV}$ above this potential, with a scan rate of $1 \mathrm{mV} \mathrm{s}^{-1}$. Data from potentiostatic polarization measurements were treated with Corr View software to obtain corrosion potential, corrosion current and polarization resistance.

\section{RESULTS AND DISCUSSION}

\section{Morphological characterization}

Figure 1 shows the SEM micrographs obtained for all systems studied MTSF, MT1F and MT2F after the process of dip-coating and UV curing.
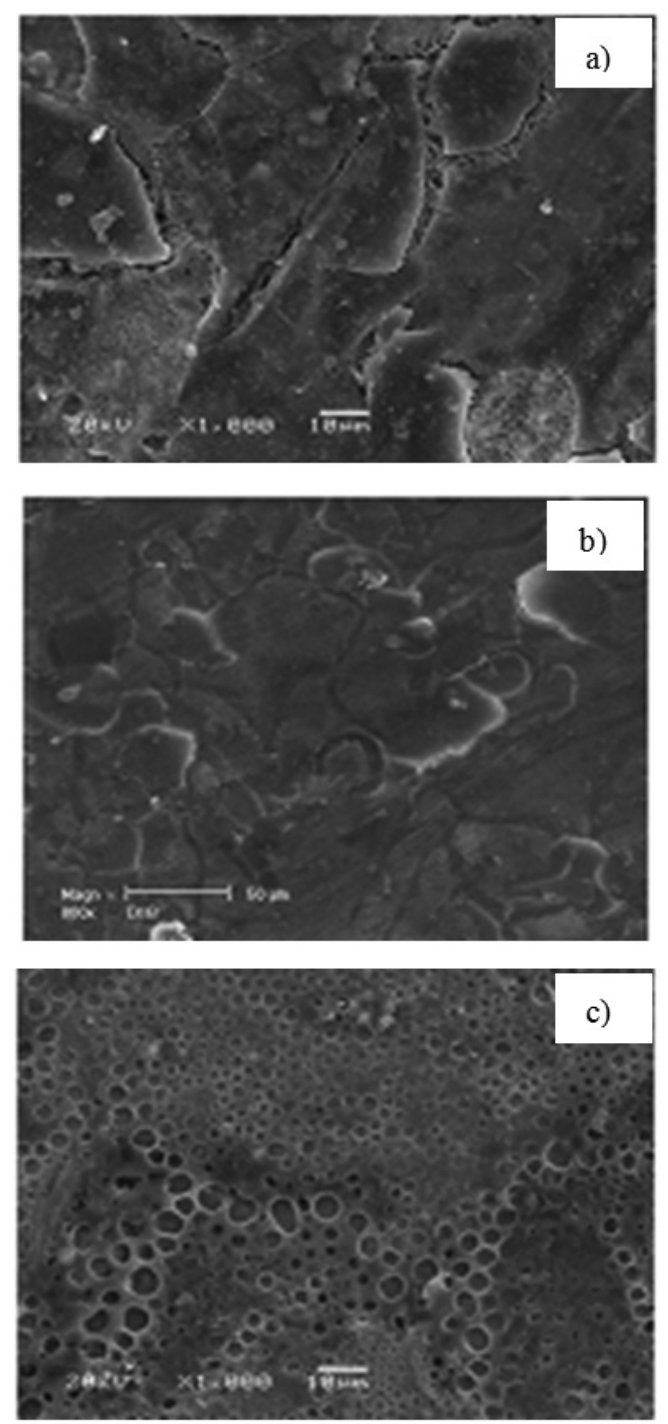

Figure 1: SEM micrographs for the systems studied: a) MTSF (b) MT1F and (c) MT2F.

It is observed in the micrographs of Figure 1 that the MTSF system (Figure 1-a) showed cracks, indicating the involvement of the adhesion of the film to the substrate. This is due to the fact that the film was exposed to UV radiation in the absence of a sensitive substance (photoinitiator) able of absorbing such radiation at a wavelength UV, thus causing degradation of the film formed. For MT1F system (Figure 1-b), the proportion of $1 \%$ of photoinitiator was not enough to absorb all UV radiation, there is still the formation of small cracks, but to a lesser extent than the system MTSF. On the other hand, for the system with $2 \%$ photoinitiator (MT2F) there was no formation of cracks in the film, only the formation of spherical discontinuities, but without compromising the adhesion of the coating to the substrate.

The layer thickness of the films was determined by analysis in cross-section and obtained by SEM (Figure 2). The results are shown in Table2.

Table 2. Layer thickness of the coatings obtained.

\begin{tabular}{|c|c|c|}
\hline Sample & Thickness $(\mu \mathrm{m})$ & Standard deviation $(\mu \mathrm{m})$ \\
\hline MTSF & 1.89 & 0.41 \\
\hline MT1F & 1.75 & 0.16 \\
\hline MT2F & 1.20 & 0.46 \\
\hline
\end{tabular}



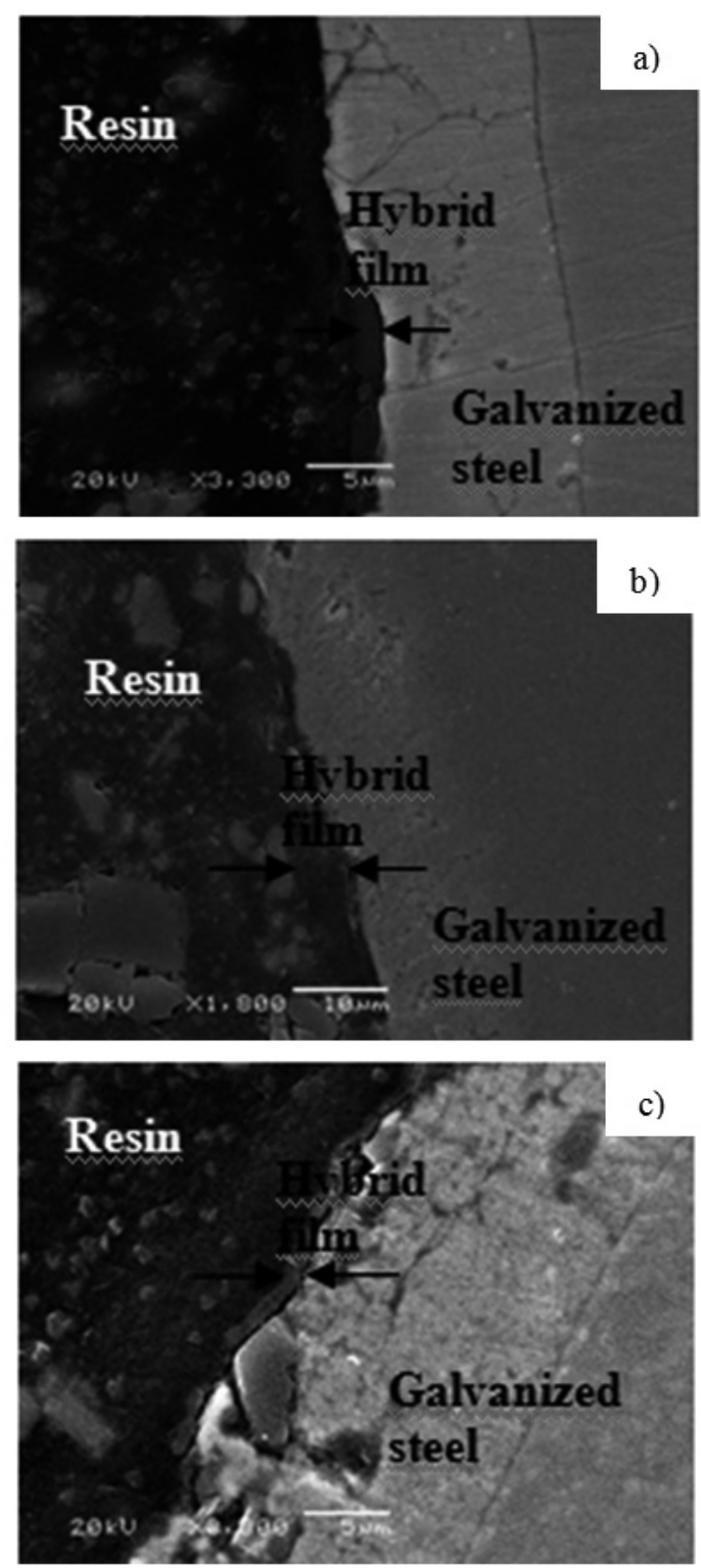

Figure 2: SEM micrographs of cross-sectional analysis for pre-treatments: a) MTSF (b) MT1F and (c) MT2F.

According Figure 2 and Table 2, the increase of the UV radiation, decreases the thickness of the layer. This is due to the fact that the excess unabsorbed radiation at a wavelength UV reaction rises "cross-linking", reducing its reactivity. This loss of reactivity is caused by the conversion of the silanol groups to siloxanes. As result, the system has a film with greater layer thickness, but with cracks. For MT2F system, in which the ultraviolet radiation was absorbed in a more intense way than the MT1F system, the curing mechanisms by free radical initiated polymerization reaction to yield a film thinner, but dense.

\section{Wettabiliy}

Figure 3 shows images obtained by the sessile drop method for determining the contact angle. From Table 3, it is possible to observe the contact angle measurements obtained for the studied systems.
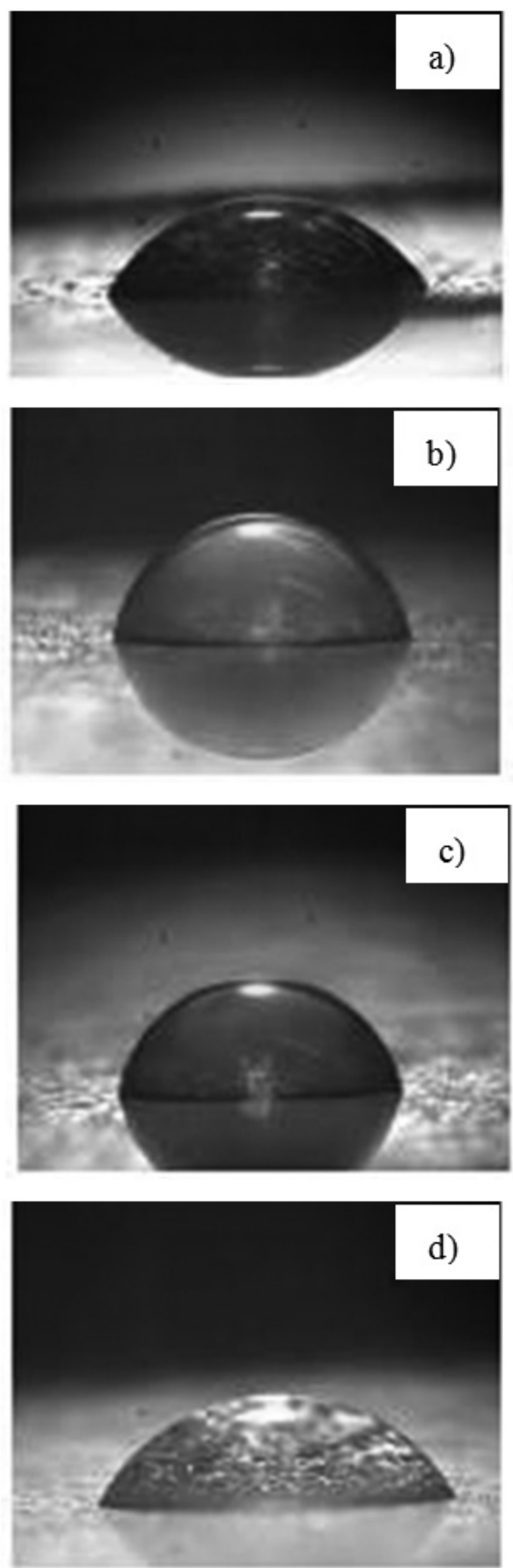

Figure 3: Images obtained for determining the contact angle for the systems studied: (a) MTSF, (b) MT1F, (c) MT2F and (d) AG. 
Table 3. Average contact angle and standard deviation.

\begin{tabular}{|c|c|}
\hline Sample & Contact angle \\
\hline AG & $66^{\circ} \pm 1.0$ \\
\hline MTSF & $65^{\circ} \pm 1.8$ \\
\hline MT1F & $80^{\circ} \pm 0.8$ \\
\hline MT2F & $80^{\circ} \pm 0.9$ \\
\hline
\end{tabular}

The contact angle results (Figure 3 and Table 3) for systems with the addition of photoinitiators MT1F (Figure 3-b) and MT2F (Figure 3-c) presented the highest values in relation to the galvanized steel without coating (Figure 3-d) and in relation to the coating without photoinitiator (Figure 3-a), which indicates that the obtained film reduced surface wettability. However, among these systems, there was no difference in results of contact angle as can be seen in Table 3. This can be associated to the concentration of the photoinitiator, because the MT1F system, despite its small cracks, has a layer thickness larger than MT2F system that had no cracks in the film formed, but the presence discontinuities. However, it can be seen that the system MTSF presented the lower value for the contact angle being very close to the value of galvanized steel without coating (Figure 3-d), which indicates that the UV cure for the system without photoinitiator was excessive, because there was a degradation of the film formed as shown by SEM (Figure 1-a).

Infrared Spectroscopy

Figure 4 shows the IR spectra of the films with precursors alkoxide of 3 (trimetoxysililpropil) methacrylate (TMSPMA) and tetraethoxysilane (TEOS) with addition of cerium nitrate in a concentration of $0.01 \mathrm{~mol}^{-\mathrm{L}^{-1}}$ and with addition of photoinitiators ( 1 and $2 \%$ ) and no photoinitiator. The films were cured by the process of UV radiation.

In Figure 4, the band with intense peak at $1020-1090 \mathrm{~cm}^{-1}$ corresponding to dense lattice formed by Si-O-Si. It can be observed that the MTSF sample, whose film was exposed to UV radiation in an excessive way, presents a higher intensity in this band than the samples containing photoinitiators (MT1F and MT2F). This indicates that the MTSF system had a better cross-linking, obtaining a greater thickness layer (Figure 2-a), but with cracks (Figure 1-a). Moreover, an intense peak is observed in this region for the MT2F system that, despite having a smaller thickness layer, has a dense film without cracks, unlike MT1F system.

However, the bands observed at $3400 \mathrm{~cm}^{-1}$ and 940 are characteristics of the axial deformation of $\mathrm{OH}$ in the $\mathrm{Si}-\mathrm{OH}$ that were not cross-linked in the process of curing. In this case, it is observed that the band is more intense to the system MT2F, where UV radiation is absorbed by the photoinitiator, which may have led to a greater amount of $\mathrm{Si}-\mathrm{OH}$ uncross-linked. This may have caused the low layer thickness for this system. On the other hand, the system MTSF got a peak of lower intensity in this region, due the increasing of the reactions of cross-linking, caused by the excessive radiation without being absorbed in the UV wavelength. This resulted in the reducing of its reactivity, caused by the conversion of silanol groups $(\mathrm{Si}-\mathrm{OH})$ to siloxane $(\mathrm{Si}-\mathrm{O}-\mathrm{Si})$. Besides, the MT1F system presented the lower peak, indicating that $\mathrm{Si}-\mathrm{OH}$ groups were crosslinked in the curing process. But, due the lower concentration of photoinitiator, this system absorbed part of the UV radiation, having the formation of a cracked film. It is observed that the band around $870 \mathrm{~cm}^{-1}$ is related to the symmetrical and asymmetrical stretching $\mathrm{CH}\left(\mathrm{CH}_{2}\right.$ and $\left.\mathrm{CH}_{3}\right)$.

\section{Open circuit potential and potentiostatic polarization}

The values of open circuit potential (Figure 5-a) for all systems studied were very close to the values of galvanized steel. This shows that all films presented cracks (MTSF and MT1F) and discontinuities (MT2F) on the surface, as shown in the SEM images (Figure 1). However, it is possible observe a shift of open circuit potential towards more negative potentials for the hybrid film with higher concentration of photoinitiators (MT2F).

From the extrapolation of Tafel slopes, applied to the polarization curves obtained (Figure 5-b), it was determined the corrosion current (icorr), corrosion potential (Ecorr), and polarization resistance ( $R p$ ) for the systems studied (Table 4). The results obtained from testing of polarization (Table 4) show that, the pretreatment with the hybrid film studied promoted the increase of polarization resistance $(\mathrm{Rp})$ and a decrease of icorr compared to uncoated galvanized steel for all systems obtained (Table 4 ) demonstrating the protective action of these pretreatments.

From the results obtained by polarization curves, it was not possible observe a significant difference in corrosion performance. However, the coating with higher concentrations of photoinitiator (MT2F) was the system that have better performance (higher value of Rp and lower icorr value) compared to other systems studied. This demonstrates the influence of the photoinitiator in the absorption by UV radiation to form the dense layer and effective against corrosion.

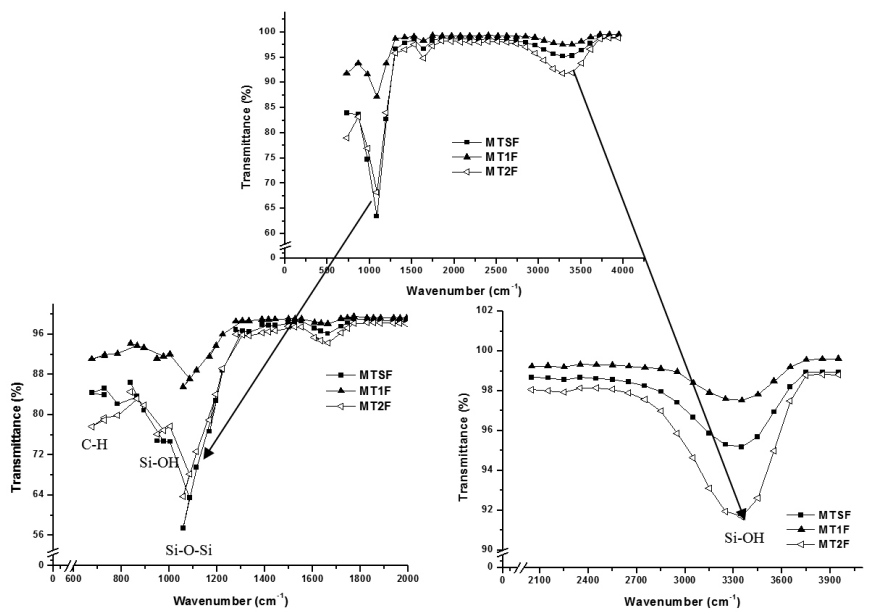

Figure 4: Infrared spectrum for the studied samples: MTSF, MT1F and MT2F. Free films solidified without the presence of substrate (free standing films).
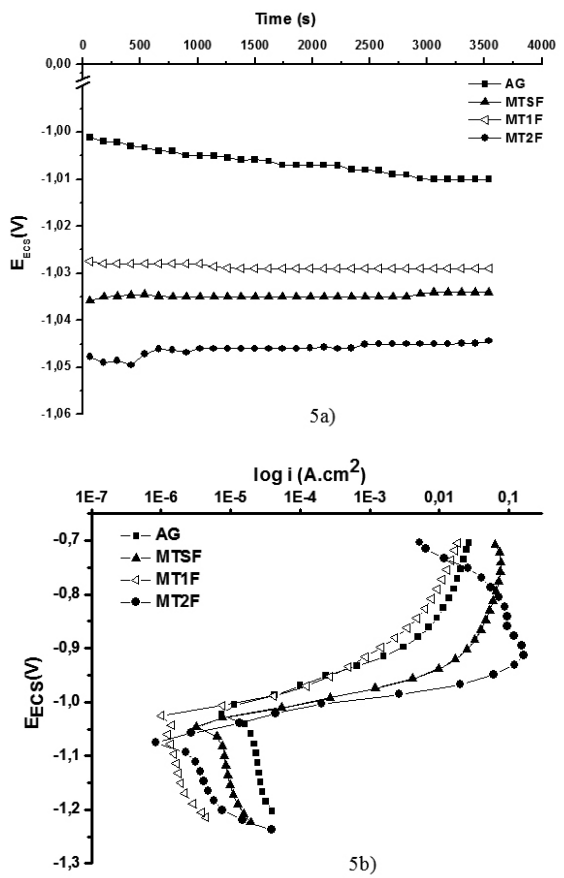

Figure 5: (a) Open circuit potential (OCP) and (b) polarization curves for all systems studied and galvanized steel without the coating.

Table 4. Values obtained from the simulation of the Tafel slopes.

\begin{tabular}{|c|c|c|c|}
\hline Samples & $\mathrm{I}_{\text {corr }}\left(\mathrm{A} . \mathrm{cm}^{2}\right)$ & $\mathrm{E}_{\text {corr }}(\mathrm{mV})$ & $\mathrm{Rp}\left(\mathrm{Ohm} . \mathrm{cm}^{2}\right)$ \\
\hline AG & $1.39 \times 10^{-4}$ & -1017 & $1.87 \times 10^{2}$ \\
\hline MTSF & $1.74 \times 10^{-5}$ & -1038 & $1.49 \times 10^{3}$ \\
\hline MT1F & $1.41 \times 10^{-5}$ & -1041 & $1.84 \times 10^{3}$ \\
\hline MT2F & $4.37 \times 10^{-6}$ & -1070 & $5.95 \times 10^{3}$ \\
\hline
\end{tabular}




\section{CONCLUSION}

The morphological analysis showed that the system MTSF presented cracking, by the fact that the film was exposed to UV radiation without the presence of a sensitive substance (photoinitiator) able of absorbing such radiation at a wavelength $\mathrm{UV}$, thus causing degradation of the film formed. Fhurtermore, the addition of $1 \%$ of the photoinitiator (MT1F) was not enough to absorb all UV radiation, with the formation of small cracks, while for the system with $2 \%$ of the photoinitiator (MT2F) there was no formation of cracks at the film, only the formation of spherical discontinuities, but without compromising the adhesion of the coating to the substrate.

Concerning the IR analysis, it can be seen that the sample MTSF, which the film was exposed to UV radiation in an excessive way, presented higher formation of $\mathrm{Si}-\mathrm{O}-\mathrm{Si}$ bond than the samples whose formulations containing photoinitiators (MT1F and MT2F). This indicates that the system MTSF got a better cross-linking, getting a thicker layer, but with cracks. Nevertheless, one can observe an intense peak in this region for the system MT2F that, although a lower layer thickness, presented a dense film without cracks unlike the MT1F system.

The results showed that the systems studied with the addition of photoinitiators, MT1F and MT2F showed to be more hydrophobic in comparison to the system without photoinitiator (MTSF). This indicates that the use of photoinitiator contributed to the polymerisation process of the coating during the UV curing. In addition, in the polarization process it was observed a behavior very similar in the coatings studied and a tendency to improve the performance concerning corrosion resistance by increasing the concentration of photoinitiator.

It is concluded that the best system is MT2F, in which ultraviolet radiation is absorbed in the curing process through free radicals that initiate polymerization reaction resulting in a dense film and without cracks.

\section{ACKNOWLEDGEMENTS}

This work was supported by CAPES, an organ of the Brazilian Government for the training of human resources. The authors also thank the financial support of CNPq and FAPERGS, Microscopy Center of UFRGS by SEM analyzes and the Laboratory of Optics \& Laser Institute of Physics of UFRGS by contact angle analysis

\section{RFERENCES}

1. Yamasaki, M. C. R., A Cura de Tintas, Vernizes e Revestimentos por Ultravioleta e Feixe de Elétrons, Associação Técnica Brasileira de Cura por Radiação, Instituto de Pesquisas Energéticas e Nucleares, São Paulo (1997).

2. Stowe, R. W., Pratical Aspects of Irradiance and Energy Density in UV Curing, Radtech 2000, Baltimore; Procedings; 536-544 (2000).

3. Pietschmann, N., UV curable metal coatings - special possibilities and problems. In: Congress Proceedings, Hannover, 8, 135-144 (2005).

4. Roose, P., I. Fallais, C. Vandermiers, M. G. Olivier and M. Poelman., Radiation curing technology: An attractive technology for metal coating. Progress in Organic Coatings, 64, 163-170 (2009).

5. EPA - Environmental Protection Agency. Toxicological review of hexavalent chromiumre (1998). Available in http://www.epa.gov. Accessed: 15/10/2011

6. Fazenda, J. M. R., Tintas \& Vernizes Ciência e Tecnologia, Abrafati, $3^{\circ}$ edition, 822-838 (2005)

7. Graen, J. H., Aplication properties of UV curable powder coatings, Radtech Europe 2003, Berlin; Procedings, 1, $45-53$ (2003).

8. Lin, A., FDA compliant testing for energy curable materials used in flexible packaging, Radtech Europe 2003, Berlin; Procedings, 1, 323-335 (2003).

9. Rahn, A. G., Free-Radical UV-Curable coatings and inks for metal and glass, Radtech 2003, Zurich (2003) 